\title{
Motion Capture and Intelligent Correction Method of Badminton Movement Based on Machine Vision
}

\author{
Yibo Zhang, Jianjun Tang, and Hui Huang $\mathbb{B}$ \\ College of Competitive Sport, Beijing Sport University, Beijing 100084, China \\ Correspondence should be addressed to Hui Huang; 2247@bsu.edu.cn
}

Received 4 June 2021; Revised 5 July 2021; Accepted 22 July 2021; Published 2 August 2021

Academic Editor: Sang-Bing Tsai

Copyright (c) 2021 Yibo Zhang et al. This is an open access article distributed under the Creative Commons Attribution License, which permits unrestricted use, distribution, and reproduction in any medium, provided the original work is properly cited.

\begin{abstract}
In recent years, badminton has become more and more popular in national fitness programs. Amateur badminton clubs have been established all over the country, and amateur badminton events at all levels have increased significantly. Due to the lack of correct medical supervision and health guidance, many people have varying degrees of injury during sports. Therefore, it is very important to study the method of badminton movement capture and intelligent correction based on machine vision to provide safe and effective exercise plan for amateur badminton enthusiasts. This article aims to study the methods of motion capture and intelligent correction of badminton. Aiming at the shortcoming of the mean shift algorithm that it is easy to lose the target when the target is occluded or the background is disturbed, this paper combines the mean shift algorithm with the Kalman filter algorithm and proposes an improvement to the combined algorithm. The improved algorithm is added to the calculation of the average speed of the target, which can be used as the target speed when the target is occluded to predict the area where the target may appear at the next moment, and it can also be used as a judgment condition for whether the target is interfered by the background. The improved algorithm combines the macroscopic motion information of the target, can overcome the problem of target loss when the target is occluded and background interference, and improves the robustness of target tracking. Using LabVIEW development environment to write the system software of the Japanese standard tracking robot, the experiment verified the rationality and correctness of the improved target tracking algorithm and motion control method, which can meet the real-time performance of moving target tracking. Experimental results show that $83 \%$ of amateur badminton players have problems with asymmetric functions and weak links. Based on machine vision technology, it can provide reliable bottom line reference for making training plans, effectively improve the quality of action, improve the efficiency of action, and promote the development of sports competitive level.
\end{abstract}

\section{Introduction}

1.1. Background. In recent years, badminton has become more and more popular in China's national fitness, the number of people participating in physical exercise more than once a week reached 720 million, and the number of people who regularly participated in physical exercise reached 425 million. The physical fitness of the masses has improved steadily. The public service system for national fitness that supports the national development goals and meets the requirements of building a well-off society in an all-round way is becoming more and more perfect. The development pattern of national fitness led by the government, coordinated by departments, and participated by the whole society has become increasingly clear. Under the guidance of the national fitness program, badminton has become one of the most popular national fitness programs in China. Badminton is becoming more and more popular among the public, and the density and intensity of exercises have reached a high level. However, without standardized medical supervision and health care guidance, many people have different degrees of sports injuries. An effective and scientific test and evaluation method can effectively screen the asymmetry and weak links of badminton enthusiasts, discover the limitations of functional sports as soon as possible, help badminton enthusiasts reduce the risk of colds, and further strengthen the stability and coordination of the action posture. 
1.2. Significance. Due to the characteristics of badminton, badminton enthusiasts need to repeatedly change speed and direction throughout the game, which puts forward high requirements on their competitive level and physical fitness level, especially the requirements for functional stability and sports flexibility higher. Studies have confirmed that long-term high-intensity repetitive exercises, lack of warm-up activities, and asymmetry in exercise techniques increase the risk of injury for badminton enthusiasts. Therefore, this research aimed at the particularity of badminton enthusiasts, applied machine vision technology to test amateur badminton enthusiasts, and designed a targeted corrective intervention program. The significance of this research is to find out the problem of badminton enthusiasts' limited movement function through effective selection, analyze its influencing factors, and formulate targeted and personalized corrective training programs to help them improve the quality of their movement patterns, so as to prevent failures, the purpose of sports injury of the injured, delaying the development of the disease, and preventing the injured from reinjury.

1.3. Related Work. In the sport of badminton, the research on sports intelligence of athletes still needs to be perfected and deepened. Lau pointed out that, in the past two decades, sports analysis of athletes' performance has been basically qualitative. However, with the latest developments in technology related to input acquisition sensors, computer hardware, and algorithms, computerized human motion analysis for athletes is becoming more and more common. In this study, a novel cost-benefit analysis reference framework is proposed to evaluate the advantages and tradeoffs of various human motion capture and analysis systems in terms of cost, ease of implementation, and expected results. The framework can also help us determine the best candidate technology for the case study of a badminton coach. The study shows that the label-free technology based on consumer level is the most feasible because it has the advantages of low startup and operating costs, minimal configuration, and portability, but it compromises the accuracy of the results [1]. Massaroni et al. proposed that a motion capture system can be used for breathing assessment. In order to calculate the change in respiratory volume over time from the marked trajectory, a geometric model is essential. This study describes a novel model that can calculate volume changes and calculate breathing parameters by using a motion capture system. The novel method, the prismbased method, calculates the volume inside the chest by defining 82 prisms from 89 marks attached to the subject's chest. The volume calculated by this method is compared with the volume of spirometry, and compared with the volume calculated by the conventional method based on the tetrahedral decomposition of the chest wall, and it is integrated into a commercial motion capture system, However, the use of this model requires the collection of a large amount of data and a lot of manpower and resources [2]. Panagiotakis et al. proposed that motion capture technology is a new technology for capturing and analyzing the $3 \mathrm{D}$ trajectory of athletes' technical movements, by capturing the athletes' movements, and then comparing them with standard movements, to help them train and provide an accurate basis for scientific training. He proposed an optimization method for basketball teaching and training system design based on motion capture technology. Comparing the simulation results with the training videos of the athletes, you can display the training actions and standard actions on the same screen, and compare and analyze the actions, but the video pixel requirements are high [3]. But it needs a lot of money and manpower.

\subsection{Main Content}

(1) It is an in-depth exploration of the current research on badminton players in my country. The current research on badminton players is mainly focused on how to improve their technical and tactical level [4] and how to improve their physical fitness. Most of these can be judged through subjective observations, which are relatively comparative, which is simple and easy. And this article attempts to study the athletes from their motion capture of playing badminton, because it involves some visual knowledge [5], which is relatively abstract and difficult to study. This article attempts to conduct exploratory research in this field.

(2) A set of evaluation system for evaluating the sports intelligence of badminton players has been formed. At present, there are few researches on the evaluation of badminton players' sports intelligence, and a relatively complete evaluation system has not been formed. This article explores the method of motion capture and intelligent correction of badminton movement based on machine vision and improves the research on the evaluation of existing badminton players. But the key lies in the continuous improvement through the intelligent correction method.

(3) It is a further improvement of the intelligent correction of existing badminton players. In the past, corrections often judged the athlete's potential or pros and cons based on the athlete's competition actions, often making wrong evaluations and misleading the coach's judgment. The index system constructed in this article [6] systematically evaluates badminton players from all aspects, and the ultimate goal is to motivate them to exercise more scientifically and reasonably.

\section{Research Methods of Motion Capture in Badminton}

\subsection{Research Methods}

2.1.1. Observation Method. The whole experiment was tracked and monitored [7], and two high-definition digital cameras were used to record; the video and photographed 
image data were used to analyze and evaluate the testing situation and intervention effects of sports enthusiasts [8].

2.1.2. Mathematical Statistics and Processing. Data results were represented as mean \pm standard deviation $(M \pm \mathrm{SD})$. We used soss 21.0 for data statistics and used paired $t$-test to analyze the difference between FMS scores and test scores before and after correction [7, 9]; $t$-test is mainly used for small sample size (e.g., $n<30$ ) and overall standard deviation $\sigma$, unknown normal distribution. $t$-test is to use t-distribution theory to infer the probability of difference, so as to compare whether the difference between two means is significant. Use independent sample $t$-test to analyze FMS scores of different genders. $P<0.05$ is statistically significant.

2.2. Moving Target Detection. Moving target detection is based on a video stream, using statistical methods to segment the foreground and background of the image, determine the location and texture characteristics of the target, and describe the moving target statically. Common target detection methods include frame difference method, optical flow method, and background difference method. The optical flow algorithm uses the feature that the brightness of the object changes at the same time when it is moving to detect the moving object. Its computational complexity is high and it is sensitive to the changes in the light in the image. In the background subtraction method, a reference background is first established, and then the current image is used for subtraction to obtain the foreground target, but it is greatly affected by changes in light and shadow, and the reference background needs to be corrected in real time as the background information changes. The interframe difference method is to make frame difference for continuous images. Compared with the optical flow method, the amount of calculation is small, and it can achieve real-time effects. It can be used in a dynamic background environment. It does not need to continuously update the reference background and is less affected by light changes [10].

2.2.1. Interframe Difference Method [11]. The frame mode (as shown $i n$ Figure 1) is an algorithm for using the image of the foreground objects and displaying dynamic different symbols. When a moving target appears in a continuous video stream, the pixel value of the moving target point has obvious changes in two adjacent frames of image. The moving target point is detected by calculating the absolute value of the pixel value difference between the two frames.

In formula (1), $T$ is the threshold set for image dynamic detection, the pixel with $D(x, y)=1$ is the dynamic target [12], and the pixel with $D(x, y)=0$ represents the static background. For this single-pixel frame difference method, due to the interference of system noise, a lot of pixel noise will be extracted as moving target points. In order to eliminate the interference of system noise, the frame difference algorithm based on sliding window is adopted (as shown in formula (2)). For the pixel $I(i, j)$, a $3 \times 3$ sliding window with the current pixel as the midpoint is established.

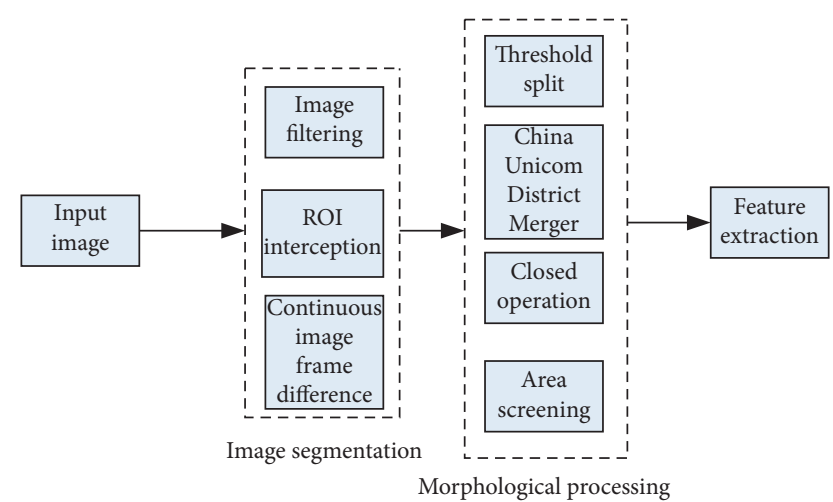

FIGURE 1: Block diagram of the algorithm for capturing badminton by frame difference method.

For each pixel, make a frame difference to the pixels in its $3 \times 3$ neighborhood. If the absolute value of the frame difference result is greater than the set threshold, then the pixel is a dynamic target; otherwise the pixel is static background.

$$
\begin{aligned}
& D(x, y)= \begin{cases}1, & \text { if }|I(t)-I(t-1)|>T, \\
0, & \text { others, }\end{cases} \\
& D(x, y)= \begin{cases}1, & |I(t)-I(t-1)|>T, \\
0, & \text { others. }\end{cases}
\end{aligned}
$$

The interframe difference method is suitable for searching the situation where the foreground is violently moving and the background is relatively stable, and the sensitivity of target detection is controlled by adjusting the threshold. The interframe difference method has a simple algorithm, and a small amount of calculation, and is insensitive to light, but it cannot detect objects that move too slowly. The detected dynamic target may also be incompletely extracted, or two foregrounds may appear. There are two suspected badminton points in the result obtained by the frame difference. In order to determine the true position of the badminton, the three-frame frame difference method is adopted, the three-frame frame difference method is to make the frame difference between the first two frames and the last two frames, respectively, and the frame difference results obtained are $Q 1$ and $Q 2$, respectively; let $Q=Q 1 \cup Q 2$; then $Q$ is the required result. After the same three-frame frame difference, there are few interference points, and there will be no repeated foreground, that is, the situation in the frame difference result of repeated high-speed moving targets. The red area is the real badminton, and the green, blue, and pink are interference points.

2.2.2. Optical Flow Method. The essence of the optical flow method is to calculate the speed and direction of the object in the field of view by calculating the brightness of all pixels in the image over time. According to the principle of visual perception, the light intensity of the objective object projected on the camera sensor $[13,14]$ plane in a short time is unchanged, so the gray value of the image does not change, that is, the principle of gray invariance. Assuming that the 
brightness of the voxel located at $(x, y, t)$ is $I(x, y, t)$, the voxel moves $(\Delta x, \Delta y)$ within $\Delta t$, so a conclusion that the brightness is the same can be obtained.

$$
I(x, y, t)=I(x+\Delta x, y+\Delta y, t+\Delta t) .
$$

Assuming that the movement is small, the formula is expanded by Taylor series to get

$$
\begin{aligned}
I(x+\Delta x, y+\Delta y, t+\Delta t)= & I(x, y, t)+{ }_{\delta 2}^{\delta 1} \Delta x+{ }_{\delta 2}^{\delta 1} \Delta y \\
& +{ }_{\delta 2}^{\delta 1} \Delta t+\text { H.O.T. }
\end{aligned}
$$

From this, the basic equation of optical flow can be obtained:

$$
-{ }_{\delta 2}^{\delta 1}=\frac{\delta 1}{\delta x} u+{ }_{\delta 2}^{\delta 1} v .
$$

The derivative of the gray value with respect to time is equal to the product of the gradient of the image gray value and the components of the optical flow along the $x$ and $y$ directions. The basic equation of optical flow is a linear equation about $(u, v)$. There are two uncertain variables. In this case, the solution of $u$ and $v$ cannot be obtained. At this time, other constraint conditions must be introduced. This kind of uncertainty is the aperture problem. Choosing different constraints leads to different calculation methods. There are four main types of optical flow algorithms: Bayesian optical flow algorithm, frequency domain-based optical flow algorithm, area-based optical flow algorithm, and gradient-based optical flow algorithm.

2.2.3. LK Optical Flow Algorithm. In order to capture the high-speed movement of badminton, a $100 \mathrm{fps}$ camera is used to capture the video stream $[15,16]$. Assuming that, in a sufficiently small area, the corresponding points on the two frames of images are linear, and the movement speed and direction of adjacent points are the same, you can get $\mathrm{N}$ linear equations, written in the form of a matrix:

$$
\mathrm{Av}=b .
$$

$$
\text { Among them, } A=\left[\begin{array}{cc}
I_{x 1} & I_{y 1} \\
I_{x 2} & I_{y 2} \\
\vdots & \vdots \\
I_{x N} & I_{y N}
\end{array}\right], v=\left[\begin{array}{c}
V_{x} \\
V_{y}
\end{array}\right], b=\left[\begin{array}{c}
-I_{t 1} \\
-I_{t 2} \\
\vdots \\
-I_{t N}
\end{array}\right] \text {. }
$$

This is an overdetermined system of equations. Through the least square method, the optimal solution is the component of the optical flow at this point in the $x$ and $y$ directions.

$$
v=\left(A^{T} A\right)^{-1} A^{T} b .
$$

The premise of the LK optical flow algorithm is to select a neighborhood window and use equation (7) to solve the problem in the neighborhood window. If the neighborhood is larger, the result obtained by the optical flow algorithm is more robust. In the neighborhood, the results obtained by the optical flow algorithm are more accurate. This is because the LK algorithm is based on the assumption that the optical flow is a fixed value in the neighborhood of the pixel. If the neighborhood is small, it is easy to meet this assumption, and the final calculation result is more realistic; the larger the neighborhood, the more the previous assumptions. This is not easy to satisfy. Especially for the target of strenuous exercise, if you want to get a higher robust result, you must enlarge the domain window.

\section{Moving Target Tracking Experiment}

3.1. Gaussian Mixture Model. Since there are often some disturbances in the background (such as waves on the water surface or leaves swaying with the wind), the Gaussian mixture model $[17,18]$ is used instead of the single Gaussian distribution model. Each pixel value in the model is the superposition of the Gaussian model. For this method, the probability of a given pixel gray value is given by the following formula:

$$
P\left(I_{s, t}\right)=\sum_{i=1}^{k} w_{i, s, t} N\left(\mu_{i, s, t}, \sum_{i, s, t}\right) .
$$

Among them, $N\left(\mu_{i, s, t}, \Sigma_{i, s, t}\right)$ is the $i$-th Gaussian model and $w_{i, s, t}$ is the weight. In order to reduce the amount of calculation, the covariance can be a diagonal matrix. The model parameters are updated as follows:

$$
\left\{\begin{array}{l}
w_{i, s, t}=(1-\alpha) w_{i, s, t-1}+\alpha M \\
\mu_{i, s, t}=(1-\rho) \mu_{i, s, t-1}+\rho I_{s, t} \\
\sigma_{i, s, t}^{2}=(1-\rho) \sigma_{i, s, t-1}^{2}+\rho d_{2}\left(I_{s, t}, \mu_{i, s, t}\right)
\end{array}\right.
$$

Among them, $\alpha$ is the user-defined learning efficiency, and $\rho$ is the second learning rate, which is defined as follows:

$$
\rho=\alpha N\left(\mu_{i, s, t}, \Sigma_{i, s, t}\right) \text {. }
$$

For unmatched models, their mean and variance remain the same, but their weights will become smaller because

$$
w_{s, t}=\alpha w_{s, t}+\alpha M
$$

If there is no model matching the pixel value, the model with the minimum weight will be replaced by a new Gaussian model with the current pixel value as the mean value and variance of 2 , and the weight allocation will be the minimum. Once each Gaussian model is updated, the $K$ weights are normalized so that their weighted sum is 1 . Then sort the new weights and select only the $H$ most reliable ones as part of the background.

$$
H=\arg \min \left(\sum_{i=0}^{h} w_{i}>\tau\right) .
$$

Among them, $\tau$ is the threshold [19]. Those pixels with variance values greater than 2.5 for the selected $\mathrm{H}$ models are marked as moving points. 
3.2. Moving Target Tracking Technology. Moving target tracking refers to the analysis of multiple videos to determine or estimate motion characteristics such as the position and speed of the target. Moving target tracking algorithms can be divided into the following categories.

3.2.1. Feature-Based Tracking. The basic idea of the feature method is to extract the features of the moving target with invariable characteristics and then find the best match in the subsequent video frame images through the search algorithm, so as to realize the tracking of the moving target.

3.2.2. Area-Based Tracking. On the basis of area tracking, the moving target is divided into different small areas, and the moving target is tracked by tracking the corresponding area. In this type of tracking method, first determine the tracking area block, and then extract the corresponding features and use related algorithms to track.

3.2.3. Tracking Based on Dynamic Contour. The contourbased tracking algorithm provides more detailed target shape and deformation information, but the contour initialization is difficult, and the robustness is not high in the case of the target motion occlusion and sudden change. The first frame is automatically initialized by an algorithm based on optical flow; Markov random theory is used to estimate posterior probability density to realize contour evolution based on color features; combine global shape information with local color information to establish a new shape model to realize shape-based adaptive contour evolution [20].

\section{Motion Capture and Intelligent Correction Analysis}

4.1. Comparison of Motion Capture Systems. The comparison of five common motion capture systems is shown in Table 1.

\subsection{Intelligent Correction Analysis}

4.2.1. Comparison of Results of Different Methods of Joint Point Estimation in the Framework. We obtained the spatial distribution of each joint point of the target human body through pose derivation. Traditional methods based on graph structure models (such as Burenius [21], etc.) generally use the maximum posterior probability position as the estimation result of the joint point, but the discretization is used in the framework of this article. Search space to simplify the calculation, so for the discrete point set, the most probable position may not coincide with the discrete point. Based on this, this paper adopts the calculation of the center position of the joint point spatial area distribution as the position estimation of the joint point. This paper uses two methods to estimate the error of each joint point under the discrete granularity of $32 * 32 * 32$ for different actions in the test set to compare the error of the estimation results. Figures 2-4 show the corresponding results.
From the above comparison results, it can be seen that the estimation result of the method of obtaining the center of the joint point distribution area has less error than the method of directly selecting the maximum probability position as the joint point estimation method. The possible reason is that after discrete points are used to represent the distribution area of joint points for discretization, the probability distribution of each joint point in the search space is not continuous, so the position with the highest probability is not necessarily that the joint point is continuous. The maximum probability position in the spatial area distribution has a certain position deviation. Another possible reason is that the initialization of the distribution area of each joint point is affected by the human pose estimation results of the twodimensional images from each perspective. The joint point position estimation from different perspectives is noisy, so the maximum probability positions of the same joint point from each perspective are inconsistent when projected into the three-dimensional space. It also leads to the uneven spatial distribution of joint points and the inconsistency between the position of the discrete point with the highest probability and the position of the center of the region. To sum up, this paper uses the joint point distribution center as the joint point position estimation method can get relatively better joint point position estimation results.

4.2.2. Comparison of the Results of the Method in This Paper under Different Granular Space [22, 23] Discretization. We use the discretization search space method to simplify the solution process of obtaining the global optimal estimation of each target human joint point under the constraints of the graph structure skeleton model. The discretization method is a method that approximately characterizes the physical quantities in continuum mechanics with a finite number of parameters. Under different granularities, the size of the formed area point set increases exponentially, and the calculation time will also increase exponentially. In order to verify the impact of different discrete granularities on the joint point estimation results, we have three types of $16 * 16 * 16$ spatial discrete point sets, $32 * 32 * 32$ spatial discrete point sets, and $64 * 64 * 64$ spatial discrete point sets in the test set. The particle size was tested. The result is shown in Figure 5, and the unit is $\mathrm{mm}$.

It can be seen from Figure 5 that in the framework of this article the granularity of the discretization of the threedimensional space region is positively correlated with the estimation result of the joint point position. It is easy to know that the finer the granularity of the discrete area, the more accurately the distribution shape of the joint points in the search space can be expressed, so as to achieve better results. At the same time, we also noticed that the increase in the granularity of $32 * 32 * 32$ is larger, while the increase in the granularity of $64 * 64 * 64$ is smaller than that of $32 * 32 * 32$. This may be due to the general proportion of the periphery of the joint point distribution. 
TABLE 1: Comparison of common capture systems.

\begin{tabular}{|c|c|c|c|}
\hline System name & Composition & Advantages & Disadvantages \\
\hline Mechanical & Joints with sensors and rigid links & $\begin{array}{l}\text { Low cost, high accuracy, good real- } \\
\text { time performance, small } \\
\text { limitations, multiobject capture }\end{array}$ & $\begin{array}{l}\text { Inconvenient operation, restrictions on } \\
\text { performers' movement }\end{array}$ \\
\hline Acoustic & $\begin{array}{l}\text { Acoustic emission source, receiving } \\
\text { equipment, and processing module }\end{array}$ & $\begin{array}{l}\text { Low price, effectively solving the } \\
\text { problem of motion occlusion }\end{array}$ & $\begin{array}{l}\text { Low accuracy and susceptible to } \\
\text { interference }\end{array}$ \\
\hline Electromagnetic & $\begin{array}{l}\text { Electromagnetic transmitter, } \\
\text { receiving and sensing equipment, } \\
\text { and data processing module }\end{array}$ & $\begin{array}{l}\text { Low price, good real-time } \\
\text { performance, fast speed, mature } \\
\text { technology }\end{array}$ & $\begin{array}{c}\text { Strict environmental requirements and } \\
\text { low sampling rate }\end{array}$ \\
\hline $\begin{array}{l}\text { Optical motion } \\
\text { capture }\end{array}$ & $\begin{array}{l}\text { High-precision camera, marking } \\
\text { point, and data processing unit }\end{array}$ & $\begin{array}{l}\text { The marking point is cheap, easy to } \\
\text { use, and limited in movement and } \\
\text { has high sampling rate }\end{array}$ & $\begin{array}{l}\text { The entire system is expensive and has a } \\
\text { large amount of postprocessing, and the } \\
\text { marking points are easily obstructed }\end{array}$ \\
\hline $\begin{array}{l}\text { Video-based } \\
\text { motion capture }\end{array}$ & Camera and data processing unit & $\begin{array}{l}\text { There are no restrictions on the } \\
\text { captured objects, low cost, and low } \\
\text { environmental requirements }\end{array}$ & $\begin{array}{l}\text { The algorithm is complex and difficult to } \\
\text { implement, and the technology is not yet } \\
\text { perfect }\end{array}$ \\
\hline
\end{tabular}

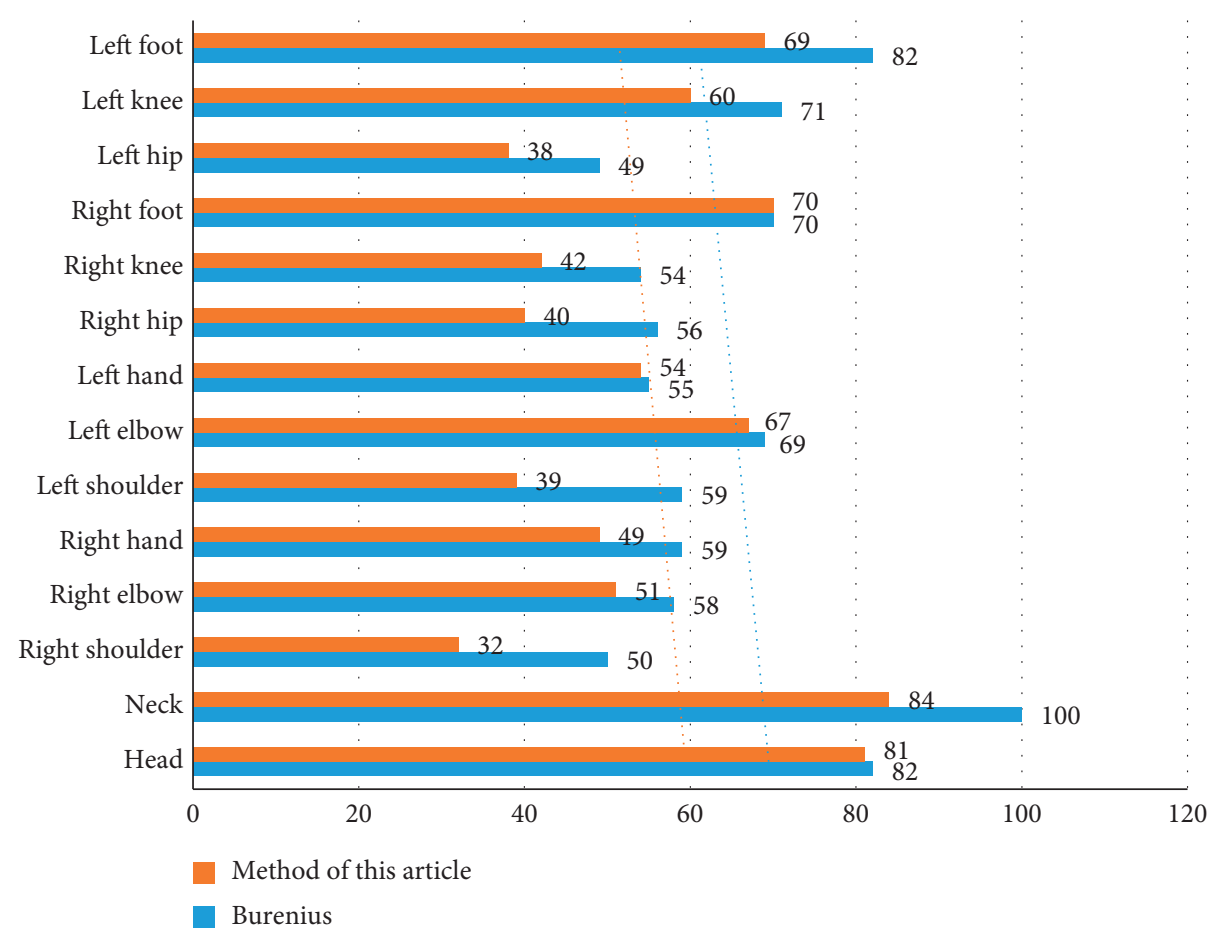

FIgURE 2: Error comparison of joint point estimation results under directions action.

The loose weight is smaller, and the finer the particle size, the greater the impact on the peripheral distribution, and the smaller the impact on the central area so the impact of the particle size on the joint point estimation results has a limit value. And the method used in this paper to obtain the joint point distribution center position as the joint point position estimation method can reduce the influence of the discrete granularity on the estimated structure to a certain extent. Since each joint point message needs to traverse all discrete points in the search space, the larger the granularity, the higher the calculation complexity of each joint point prediction, and the calculation time of the overall human pose estimation is also a series [24]. Considering the balance of calculation speed and result accuracy, the maximum granularity of the experiment is $64 * 64 * 64$, and the optimal joint position estimation result can be obtained under the discrete granularity of $64 * 64 * 64$.

\subsection{Situation before and after the Action Correction Intervention of Badminton Enthusiasts of Different Genders}

4.3.1. FMS Score before Correction Intervention. Analyze the difference between the preintervention score and the FMS total score (see Table 2). FMS refers to functional movement screening. The results showed that the total score of female FMS was higher than that of male, but there was no significant difference; female hurdle performance was significantly higher than that of male $(P=0.001)$; male hurdle 


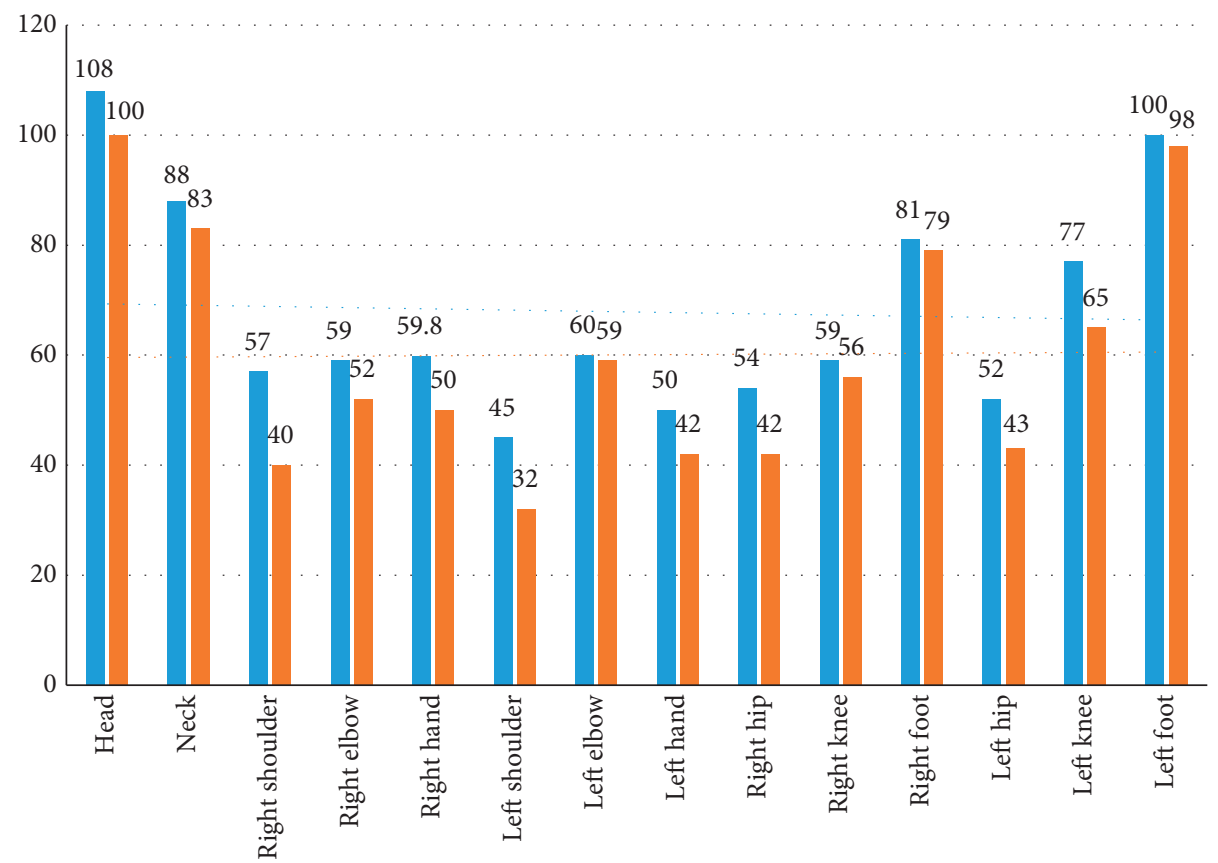

Burenius

Method of this article

FIgURE 3: Error comparison of joint point estimation results under discussion action.

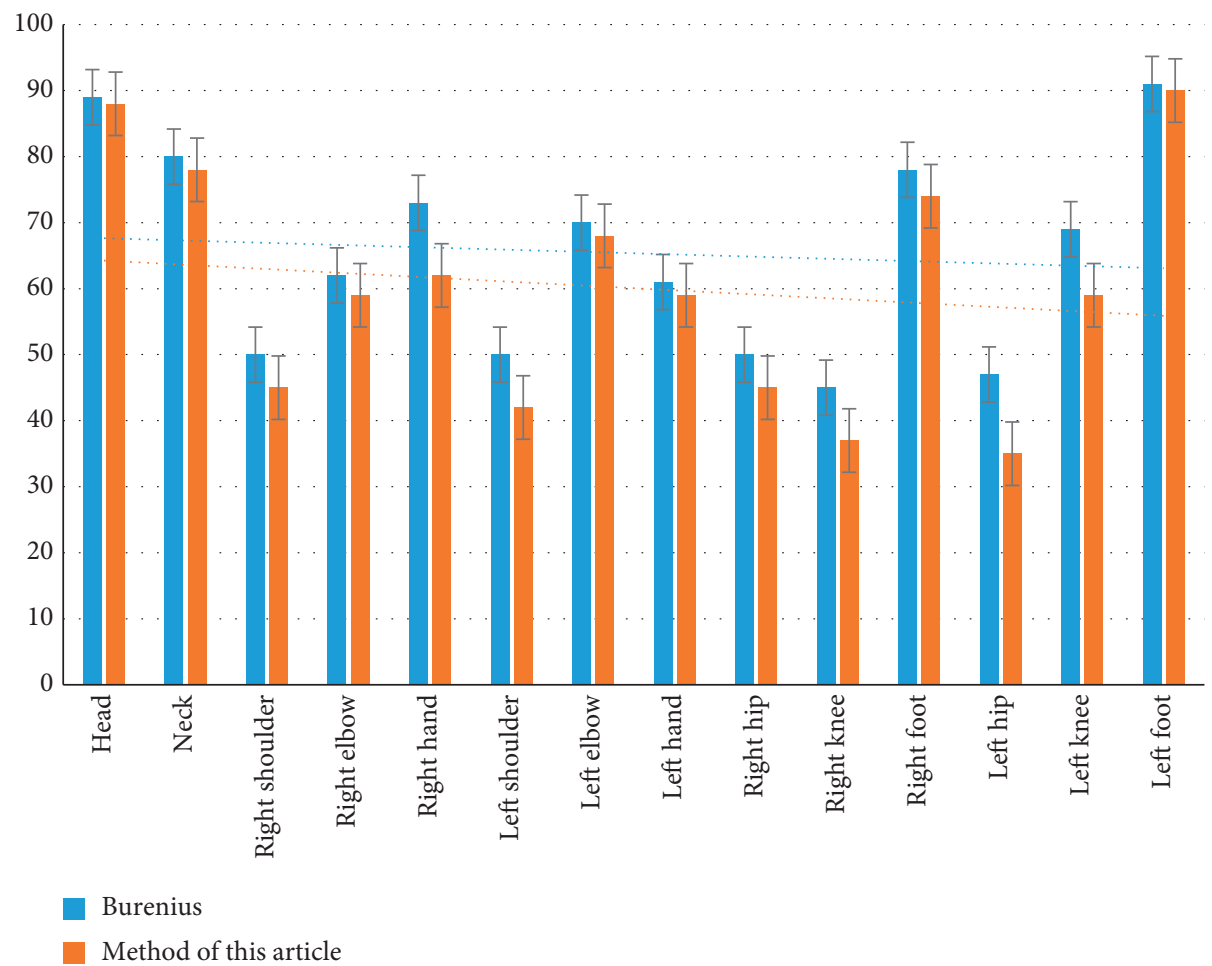

FIGURE 4: Error comparison of joint point estimation results under photo action.

performance was significantly higher than that of female $(P=0.020)$; for amateur badminton enthusiasts of different genders, there were no significant differences in squatting, lunge squatting, shoulder flexibility, and FMS scores. Only the scores of the hurdle test and the trunk stability bow test are significantly different. 


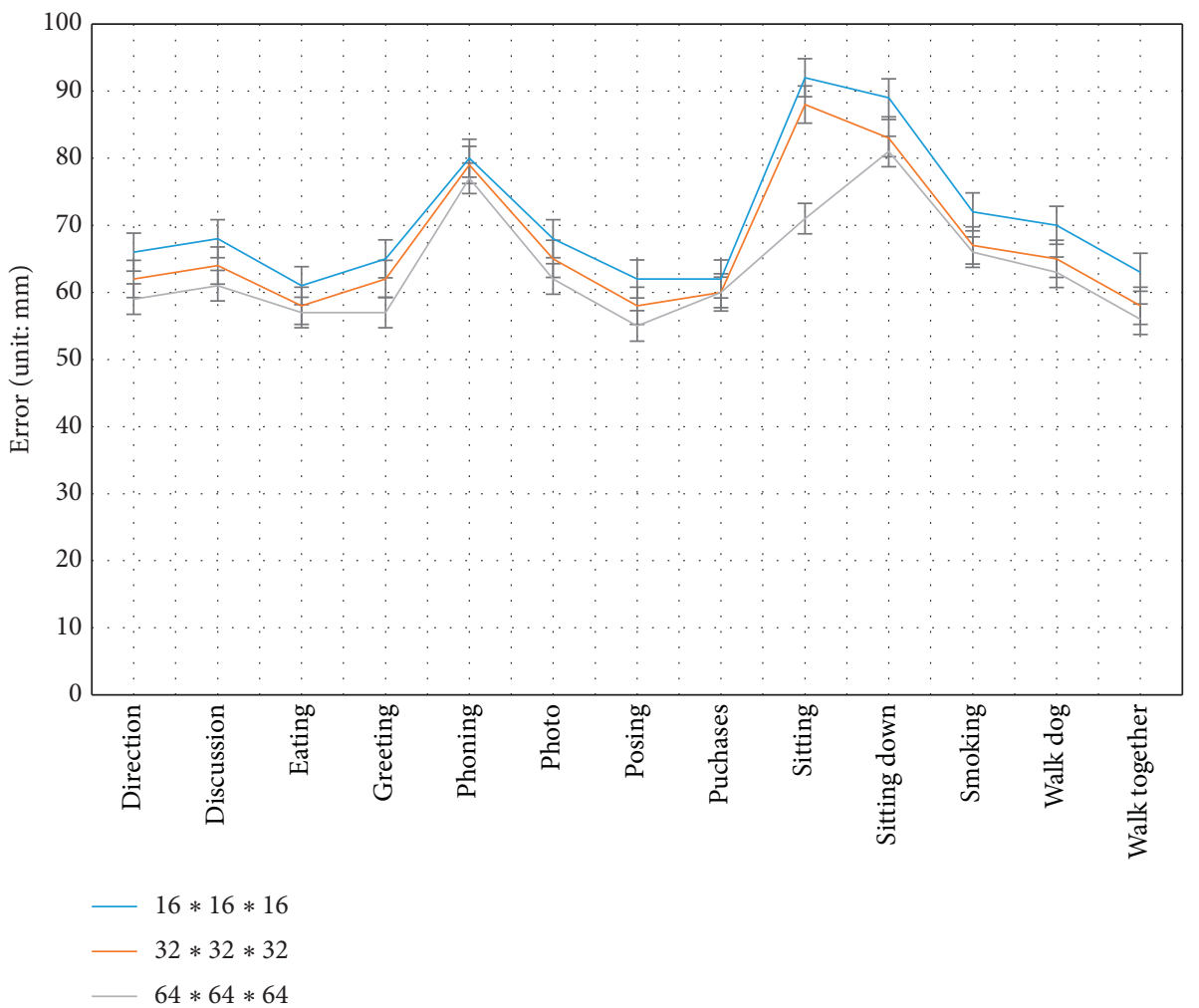

FIGURE 5: Error comparison of joint point estimation results under different discrete granularities.

TABLE 2: Comparison of FMS total scores and various test scores of sports enthusiasts of different genders before the intervention $(M \pm S d)$.

\begin{tabular}{|c|c|c|c|c|}
\hline Action mode & Male $(N=15)$ & Female $(N=9)$ & Total $(N=24)$ & $P$ value \\
\hline Squat & $1.73 \pm 0.734$ & $1.78 \pm 0.833$ & $1.75 \pm 0.737$ & 0.890 \\
\hline Hurdle & $1.13 \pm 0.352$ & $1.78 \pm 0.441^{* *}$ & $1.38 \pm 0.495$ & 0.001 \\
\hline Straight lunge squat & $1.73 \pm 0.458$ & $1.89 \pm 0.333$ & $1.79 \pm 0.415$ & 0.386 \\
\hline Shoulder flexibility & $1.67 \pm 0.488$ & $2.00 \pm 0.707$ & $1.79 \pm 0.588$ & 0.185 \\
\hline Active straight leg lift & $1.73 \pm 0.704$ & $2.00 \pm 0.500$ & $1.83 \pm 0.637$ & 0.332 \\
\hline Trunk stability push-ups & $2.47 \pm 0.516$ & $1.78 \pm 0.833^{*}$ & $2.21 \pm 0.721$ & 0.020 \\
\hline Rotational stability & $1.33 \pm 0.724$ & $1.11 \pm 0.333$ & $1.25 \pm 0.608$ & 0.318 \\
\hline FMS total score & $11.80 \pm 1.699$ & $12.56 \pm 0.882$ & $12.08 \pm 1.472$ & 0.231 \\
\hline
\end{tabular}

Note. The independent sample $t$-test is used to compare the FMS scores of different genders; “*” and “**”, respectively, indicate $P<0.05$ and $P<0.01$.

4.3.2. Analysis of FMS Score after Intervention. Analyze the difference between the preintervention score and the FMS total score (see Table 3). The results showed that the total score of female FMS was higher than that of male, but there was no significant difference; female hurdle performance was significantly higher than that of male $(P=0.001)$; male hurdle performance was significantly higher than that of female $(P=0.020)$; for amateur badminton enthusiasts of different genders, there were no significant differences in squatting, lunge squatting, shoulder flexibility, straight leg elevation, rotational stability, and FMS scores. Only the scores of hurdle test and torso stability bow test were significantly different.

4.3.3. Comparative Analysis of Asymmetric Changes before and after Correction Intervention. In the FMS test, five test measures may measure left and right side symmetry of the body; the purpose of these five movements is to test the functional flexibility and stability of the hip, knee, ankle joint, and shoulder joint; strength, flexibility, muscle dilation, and neuromuscular coordination of upper and lower limbs were studied. Functional flexibility and stability: test the strength, flexibility, and stretchability of upper and lower limb muscle groups and the ability of neuromuscular coordination and control. From the test results before and after the correction intervention, it was found that the subjects generally had left and right asymmetry problems. In the hurdles, lunges, shoulder flexibility, straight leg lifts, and rotation stability tests, the percentages of asymmetry were as follows: $58.33 \%, 79.17 \%, 70.83 \%, 29.17 \%$, and $37.50 \%$; after 8 weeks of corrective intervention training on the subjects, the percentage of asymmetry has improved; especially the gap between the lowest score and the highest score has narrowed. Low score actions have been significantly improved (see Table 4). 
TABLE 3: Comparison of FMS total scores and various test scores of sports enthusiasts of different genders before the intervention $(M \pm S d)$.

\begin{tabular}{lccc}
\hline Action mode & Male $(N=15)$ & Female $(N=9)$ & Total $(N=24)$ \\
\hline Squat & $2.13 \pm 0.640$ & $2.11 \pm 0.833$ & $2.13 \pm 0.680$ \\
Hurdle & $1.33 \pm 0.488$ & $2.00 \pm 0.500^{* *}$ & $1.60 \pm 0.588$ \\
Straight lunge squat & $1.80 \pm 0.414$ & $2.11 \pm 0.333$ & $1.91 \pm 0.408$ \\
Shoulder flexibility & $1.93 \pm 0.458$ & $2.11 \pm 0.601$ & $2.00 \pm 0.511$ \\
Active straight leg lift & $1.80 \pm 0.561$ & $1.78 \pm 0.441$ & $1.79 \pm 0.509$ \\
Trunk stability push-ups & $2.60 \pm 0.507$ & $2.22 \pm 0.441^{*}$ & $2.45 \pm 0.509$ \\
Rotational stability & $1.60 \pm 0.632$ & $1.67 \pm 0.500$ & $1.58 \pm 0.359$ \\
FMS total score & $13.13 \pm 1.81$ & $13.89 \pm 1.054$ & $13.42 \pm 1.59$ \\
\hline
\end{tabular}

Note. The independent sample $t$-test is used to compare the FMS scores of different genders; “*” and “**”, respectively, indicate $P<0.05$ and $P<0.01$.

TABLE 4: Asymmetry changes before and after training $(\%)(M \pm S d)$.

\begin{tabular}{|c|c|c|c|c|}
\hline \multirow{2}{*}{ FMS } & \multicolumn{2}{|c|}{ Before intervention } & \multicolumn{2}{|c|}{ After intervention } \\
\hline & $N$ & $\%$ & $N$ & $\%$ \\
\hline Hurdle & 14 & 58.33 & 5 & 20.83 \\
\hline Straight lunge squat & 19 & 79.17 & 15 & 62.50 \\
\hline Shoulder flexibility & 17 & 70.83 & 9 & 37.50 \\
\hline Active straight leg lift & 7 & 29.17 & 7 & 29.17 \\
\hline Rotational stability & 9 & 37.50 & 4 & 16.67 \\
\hline
\end{tabular}

\section{Conclusions}

With the development of modern science and technology, whether in work, daily life, or entertainment, people increasingly like to improve their physical fitness through various sports. For badminton, a sport that is widely loved by the people, there is an urgent need for badminton motion capture and intelligent correction methods in China. This is of great help to correct badminton movements and those who love to play badminton. This article first analyzes the current situation of badminton at home and abroad. Secondly, the paper analyzes the popularity of ball training abroad and whether the badminton players who are more common in China can apply their knowledge of sports theory and various skills and tactics in the brain and body. In the communication, they can well control the ontology to actively participate in sports training and competition according to the characteristics of their mental and cognitive structure. In the process of badminton training, we should pay attention to the principle of gradual and orderly progress. According to the age, gender, personality, and other characteristics of the athletes, we should also implement targeted training and correct them, so as to teach students in accordance with their aptitude, make progress together, and improve their various items index. In addition to attaching importance to indicators with high weight values, attention should also be paid to indicators that are not high, because these indicators are also part of the whole and sometimes play a key role in the process of playing, so in order to allow athletes to play their best we must pay attention to the coordination and cooperation of various indicators, so that they can be optimized and play the greatest role.

\section{Data Availability}

The data underlying the results presented in the study are available within the manuscript.

\section{Conflicts of Interest}

The authors declare that they have no conflicts of interest.

\section{References}

[1] B. Y. S. Lau, H. Y. Ting, and Y. W. D. Tan, "Cost-benefit analysis reference framework for human motion capture and analysis systems," Advanced Science Letters, vol. 24, no. 2, pp. 1249-1253, 2018.

[2] C. Massaroni, E. Cassetta, and S. Silvestri, "A novel method to compute breathing volumes via motion capture systems: design and experimental trials," Journal of Applied Biomechanics, vol. 33, no. 5, pp. 361-365, 2017.

[3] C. Panagiotakis, K. Papoutsakis, and A. Argyros, "A graphbased approach for detecting common actions in motion capture data and videos," Pattern Recognition, vol. 79, pp. 1-11, 2018.

[4] C. L. Hung, M. H. Hung, C. Y. Chang, H. H. Wang, C. S. Ho, and K. C. Lin, "Influences of lateral jump smash actions in different situations on the lower extremity load of badminton players," Journal of Sports Science \& Medicine, vol. 19, no. 2, pp. 264-270, 2020.

[5] J. Sedmidubsky, P. Elias, and P. Zezula, "Effective and efficient similarity searching in motion capture data," Multimedia Tools and Applications, vol. 77, no. 10, pp. 12073-12094, 2018.

[6] M. A. Khan, "An efficient algorithm for compression of motion capture signal using multidimensional quadratic Bézier curve break-and-fit method," Multidimensional Systems and Signal Processing, vol. 27, no. 1, pp. 121-143, 2016.

[7] Z. M. Fikri, R. H. Hambali, and K. S. Rahayu, "Validity and reliability of vicon motion capture camera over the traditional anthropometric method," Malaysian Journal of Public Health Medicine, vol. 18, no. 2, pp. 142-151, 2018.

[8] S. Ding, S. Qu, Y. Xi, and S. Wan, "A long video caption generation algorithm for big video data retrieval," Future Generation Computer Systems, vol. 93, pp. 583-595, 2019.

[9] M. C. Brunkan, "Relationships of a circular singer arm gesture to acoustical and perceptual measures of singing," Update: 
Applications of Research in Music Education, vol. 34, no. 3, pp. 56-62, 2016.

[10] L. Sun, "Research on classification and recognition of badminton batting action based on machine learning," Journal of Intelligent and Fuzzy Systems, vol. 37, no. 3, pp. 1-12, 2019.

[11] W. S. Qureshi, A. Payne, K. B. Walsh et al., "Machine vision for counting fruit on mango tree canopies," Precision Agriculture, vol. 17, no. 3, pp. 1-21, 2016.

[12] A. A. Robie, K. M. Seagraves, S. E. R. Egnor, and K. Branson, "Machine vision methods for analyzing social interactions," Journal of Experimental Biology, vol. 220, no. 1, pp. 25-34, 2017.

[13] S. Ghosal, D. Blystone, A. K. Singh, B. Ganapathysubramanian, A. Singh, and S. Sarkar, "An explainable deep machine vision framework for plant stress phenotyping," Proceedings of the National Academy of Sciences, vol. 115, no. 18, pp. 4613-4618, 2018.

[14] K. Shankar, M. Elhoseny, S. K Lakshmanaprabu et al., "Optimal feature level fusion based ANFIS classifier for brain MRI image classification," Concurrency and Computation: Practice and Experience, vol. 32, no. 1, p. 24887, 2020.

[15] A. Yang, X. Gao, and M. Li, "Design of apochromatic lens with large field and high definition for machine vision," Applied Optics, vol. 55, no. 22, pp. 5977-5985, 2016.

[16] Y. Ding, X. Zhang, and R. Kovacevic, "A laser-based machine vision measurement system for laser forming," Measurement, vol. 82, pp. 345-354, 2016.

[17] M. M. Sofu, O. Er, M. C. Kayacan, and B. Cetişli, "Design of an automatic apple sorting system using machine vision," Computers and Electronics in Agriculture, vol. 127, no. C, pp. 395-405, 2016.

[18] S. Cubero, W. S. Lee, N. Aleixos, F. Albert, and J. Blasco, "Automated systems based on machine vision for inspecting citrus fruits from the field to postharvest-a review," Food and Bioprocess Technology, vol. 9, no. 10, pp. 1623-1639, 2016.

[19] S. Wan, Y. Xia, L. Qi, Y. H. Yang, and M. Atiquzzaman, "Automated colorization of a grayscale image with seed points propagation," IEEE Transactions on Multimedia, vol. 22, 2020.

[20] R. Rajashekar and B. M. Rajaprakash, "Development of a model for friction stir weld quality assessment using machine vision and acoustic emission techniques," Journal of Materials Processing Technology, vol. 229, pp. 265-274, 2016.

[21] H. Lideskog and M. Karlberg, "Simulated continuous mounding improvements through ideal machine vision and control," Image \& Vision Computing, vol. 28, no. 12, pp. 1645-1658, 2016.

[22] T. Hachaj, M. R. Ogiela, and K. Koptyra, "Human actions recognition from motion capture recordings using signal resampling and pattern recognition methods," Annals of Operations Research, vol. 265, no. 2, pp. 1-17, 2016.

[23] J. Iqbal, M. S. Sidhu, and M. Ariff, "AR oriented pose matching mechanism from motion capture data," International Journal of Engineering \& Technology, vol. 7, no. 4, pp. 294-298, 2018.

[24] A. Suraj, K. Manoj, Q. Zhang et al., "Automated detection of branch shaking locations for robotic cherry harvesting using machine vision," Robotics, vol. 6, no. 4, p. 31, 2017. 